\title{
INVESTIGATION OF FLOW SEPARATION CONTROL BY NANOSECOND PULSED DIELECTRIC BARRIER DISCHARGE ACTUATORS
}

\author{
N. Grech ${ }^{1}$, P. Leyland ${ }^{1}$, Ph. Peschke ${ }^{2}$, and P. Ott ${ }^{2}$ \\ ${ }^{1}$ GR-SCI-IAG, IGM \\ ${ }^{2}$ GR-SCI-GTT, IGM \\ Station 9, Ecole Polytechnique Fédérale de Lausanne (EPFL) \\ Lausanne CH-1015, Switzerland
}

\begin{abstract}
The ability of nanosecond pulsed dielectric barrier discharge (DBD) actuators to control flow separation was investigated on a NACA (National Advisory Committee for Aeronautics) 0015 profile for velocities up to $24 \mathrm{~m} / \mathrm{s}(\operatorname{Re}=230,000)$. The optimal location for the actuator was determined from oil flow experiments. Moderate voltage levels were applied ( 3 and $6 \mathrm{kV}$ ) and the actuator was operated at frequencies ranging from 0.058 to $10 \mathrm{kHz}$ in pulse and burst modes. The peak effectiveness of the actuator occurred at reduced frequency values of around 1. Plasma influence was observed at all tested angles of attack (up to $26^{\circ}$ ) and the stall angle was delayed by $8^{\circ}$.
\end{abstract}

\section{NOMENCLATURE}

$\begin{array}{ll}c & \text { model chord length }[\mathrm{mm}] \\ C_{L} & \text { coefficient of lift } \\ C_{p} & \text { coefficient of pressure } \\ C_{p_{\mathrm{ss}}} & \text { coefficient of pressure suction side } \\ f & \text { frequency of actuation }[\mathrm{Hz}] \\ F_{c}^{+} & \text {reduced frequency based on model chord }\left(F_{c}^{+}=f_{c} / U_{\infty}\right) \\ n & \text { number of pulses in a burst } \\ p_{s} & \text { pressure side of the airfoil } \\ \mathrm{Re} & \text { Reynolds number based on model chord } \\ s & \text { model span }[\mathrm{mm}] \\ \mathrm{ss} & \text { suction side of the airfoil } \\ \mathrm{Tu} & \text { turbulence level }[\%] \\ U & \text { magnitude of the applied voltage }[\mathrm{kV}] \\ U_{\infty} & \text { free-stream velocity }[\mathrm{m} / \mathrm{s}] \\ \alpha & \text { angle of attack }\left[{ }^{\circ}\right]\end{array}$

This is an Open Access article distributed under the terms of the Creative Commons Attribution License 4.0, which permits unrestricted use, distribution, and reproduction in any medium, provided the original work is properly cited. 


\section{INTRODUCTION}

The ability to manipulate a flow field to improve the efficiency and performance of airfoils is of great technological importance. Efficient flow control devices can be used to modify the laminar-turbulent transition inside the boundary layer, to prevent separation, to reduce drag, and to enhance airfoil lift. They may also be used to stabilize and mix airflow in order to reduce unsteadiness which generates unwanted vibrations, noise, and energy losses [1-3].

Both active and passive flow control devices can be used to control the flow over an airfoil. Passive techniques for flow control modify a flow without any external energy expenditure such as geometric shaping to manipulate the pressure gradient and the use of fixed mechanical vortex generators for separation control or segmented leading edge slats. Though these devices are efficient in augmenting the lift, they create significant increases in mechanical complexity, manufacturing cost, and weight and can induce a parasitic drag $[4,5]$.

There are several mechanisms by which active flow control techniques act; they include specifically aligned vortex generation or wall jets and the introduction of additional momentum or energy into the flow. A localized periodic excitation close to potential separation locations can be used to impart global changes to separated flow fields, typically by flow perturbation. These perturbations can be achieved by various methods, most often by means of piezoelectric, electromagnetic, and electrostatic devices which are operated by an electromechanical driver. Compared to passive control techniques, these devices offer a significant reduction in weight, mechanical complexity, and parasitic drag; however, they possess limited bandwidth and are subject to mechanical failure as the driver is operated at resonance in order to meet the amplitude requirements necessary at flight speeds [6-8].

In the course of the past decade, the use of plasma actuators for active flow control has been implemented by many researchers and has become one of the most booming realms of aerodynamics [9-11]. Dielectric barrier discharge actuators are composed of at least two electrodes separated by a dielectric material between which a high voltage is applied creating a plasma sheet [11-13]. Various electrode geometries are possible and different signals can be used to excite the actuator. Flow control with plasma is appealing as the actuators used are entirely surface mounted, lack any mechanical parts, and possess high bandwidth while requiring relatively low power to be actuated $[9,14]$. The response time is very short enabling real-time flow control. The effectiveness of DBD actuators has been proven for velocities up to $\mathrm{Ma}=0.75$, with different geometries or actuation signals showing superiority in different flow regimes [15-18]. Two types of plasma generation are typically used for DBD actuators: Alternating current power supplies, providing a sinusoidal signal to the electrode setups, for which the dominant effects are the presence of a low-speed ionic wind by the plasma in the near-wall region, with injection of momentum in the boundary layer like 
a wall-jet and, on the other hand, nanosecond pulsed power signals that generate a localized injection of energy, a small vortex generation, and a pulsing microshock wave $[9,11]$.

The actuation frequency is typically represented by the reduced frequency $F_{c}^{+}$. A preference for low-frequency actuation is generally seen, with maximum flow attachment obtained in the range of $F_{c}^{+}=1$ to 2 for a NACA 0015 airfoil $[7,19]$. As the value of $F_{c}^{+}$is increased, the influence of the plasma on the flow attachment weakens. Two mechanisms of separation flow control have been reported as being effective in a nanosecond DBD (NS-DBD) actuators. The first mechanism operates at high frequency and is connected to the instability of the boundary layer and its turbulization. The second mechanism corresponds to the instability of the separation shear layer and the effect on its shedding structures and operates at lower frequencies. The excitation of these structures induces efficient entrainment of the flow and induces the most effective flow attachment. If the discharge power is not sufficiently large to effectively excite the shear layer, only the first mechanism remains [15,19-23]. These shedding structures have been observed also in particle image velocimetry (PIV) measurements for relatively low-speed flow (up to $60 \mathrm{~m} / \mathrm{s}$ ) in a study by Little et al. [19].

This paper details the investigation of the influence of an asymmetric NSDBD actuator on the aerodynamic characteristics of a NACA 0015 airfoil profile. The experiments were performed in a low-speed wind tunnel at inlet flow speeds of 17.4 and $25 \mathrm{~m} / \mathrm{s}$ at the angles of attack ranging from $5^{\circ}$ to $26^{\circ}$. The pressure taps along the profile chord located at mid-span on both the suction and pressure sides of the airfoil were used also to determine the baseline flow field with and without the installed actuator. Oil flow visualization experiments were used to investigate the development of the baseline flow over the suction side of the profile in order to determine the most promising location for the NS-DBD actuator. Hot wire anemometry was performed with the aim of identifying dominant frequencies in the separated shear layer over the profile. Once the actuator was installed, the influence of the plasma on the flow at pre- and poststall angles of attack at different flow speeds was investigated. Nanosecond pulsed and burst mode signals were used to actuate the plasma at different breakdown voltages and over a wide range of frequencies from $58 \mathrm{~Hz}$ to $10 \mathrm{kHz}$. Here, the influence of each of the tested variables on the induced flow attachment is analyzed.

\section{EXPERIMENTAL SETUP}

\subsection{Model}

The model used throughout the experiments is a NACA 0015 profile, with a chord length of $150 \mathrm{~mm}$, and a span of $450 \mathrm{~mm}$. It incorporates a recess $0.8 \mathrm{~mm}$ deep around the leading edge, stretching from $x / c=0.09$ on the suction side to 0.11 on 


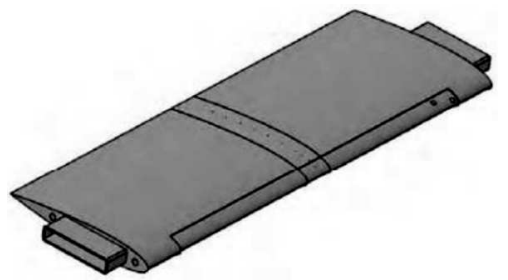

(a)

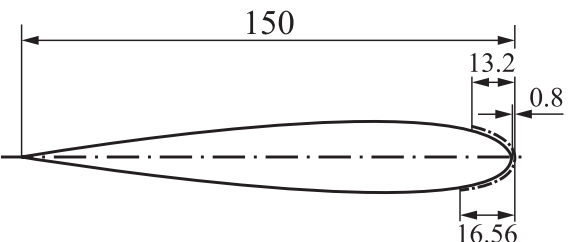

(b)

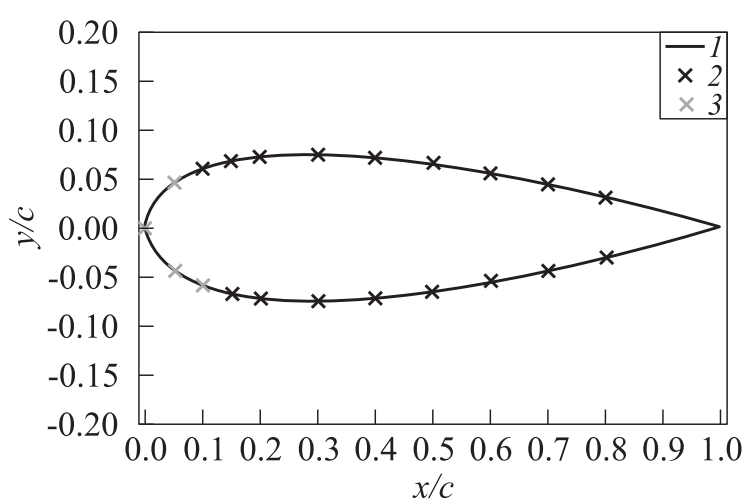

$(c)$

Figure 1 A three-dimensional view of the model used with a NACA 0015 profile $(a)$ and a cross section of the model showing the recess in the profile (dimensions are in millimeters) (b); and the NACA 0015 profile (1) with the marked pressure tap locations used (2); the grey ones (3) were blocked by the actuator

the pressure side. Its length along the span of the model is $360 \mathrm{~mm}$. This recess allowed for flush actuator integration so as to minimize surface discontinuities during the plasma experiments. Twenty one pressure taps were incorporated into the model at locations shown in Fig. 1. Only 17 of them could be used as 4 of the taps were located within the recessed area that was covered during the experiments on the model by the actuator upper electrode. The first experiments performed had the aim of characterizing the baseline flow over the model; so, the recess was filled by means of a plastic sheet whereas in the plasma experiments, the recess was covered by the DBD actuator exposed electrode.

Two Scanivalve DSA3217 pressure sensors were used to measure the static pressure from the 17 operational pressure taps along the model chord. The pressure was sampled at a frequency of $20 \mathrm{~Hz}$ and averaged over a sample of 100 measurements. The static and total pressures of the free-stream flow were measured from the pitot-tube by two Druck LPM 5480 pressure sensors sampled at a frequency of $50 \mathrm{~Hz}$ taking a sampling size of 150 measurements. 


\subsection{Wind Tunnel}

The experiments were conducted in an open-loop wind tunnel consisting of a modified radial blower driven by a frequency converter controlled by a 5.5-kilowatt asynchronous motor and connected to an asymmetric wide angle diffuser via a vibration absorber. The air was aspirated in from the laboratory into the tunnel through a dust filter, located in front of the radial blower intake, before eventually being returned to the room via a diffuser. Five screens in the wide angle diffuser prevented the flow separation. In the settling chamber, a honeycomb, dust filter, and final screen were installed to smooth out flow inhomogeneities. The modular test section had a cross section of 450-millimeter width and 300-millimeter height. A thermocouple and pitot-static tube were located upstream of the model. The angle of attack of the model was varied by rotating two circular windows fitted into the sides of the test section and was measured by means of a Bevel Box remote angle sensor with a resolution of $0.1^{\circ}$.

The turbulence level in the wind tunnel was measured by a Dantec MiniCTA $54 \mathrm{~T} 42$ hot wire anemometer. At all measured flow velocities, the turbulence level was less than $0.3 \%$.

\subsection{Dielectric Barrier Discharge Actuator Construction}

The DBD actuator was constructed from two copper electrodes separated by a dielectric layer and arranged in an asymmetric manner as shown in Fig. 2. There is a slight overlap between the upper and lower electrodes in order to encourage uniform plasma generation [10]. The encapsulated electrode has a width of $10 \mathrm{~mm}$ while the exposed electrode is $4 \mathrm{~mm}$ wide. The length of the actuator over the model span, which corresponds to the length of the exposed electrode, is $300 \mathrm{~mm}$. The dielectric barrier is composed of four layers of Kapton tape. Each layer has a thickness of $70 \mu \mathrm{m}$ : 25-micron Kapton with a 45-micron adhesive layer. The plasma forms in the upstream direction over the encapsulated

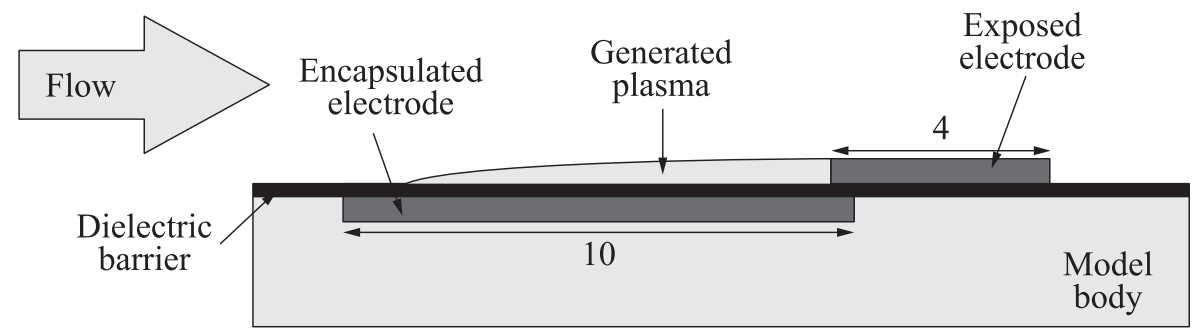

Figure 2 Scheme of the DBD actuator. Dimensions are in millimeters 
electrode as seen in Fig. 2. Thus, the first disturbance encountered by the flow is the plasma itself. The upstream edge of the exposed electrode was located at a distance of $4.5 \mathrm{~mm}$ along the chord from the leading edge of the model (at $x / c=0.03$ ). This position corresponds to the separation location as determined from oil flow visualization results given in subsection 3.1. As the occurrence of plasma on an airfoil surface induces only a relatively small effect on the airflow, it is clear that to gain any significant advantage from its use, considerable leverage should be provided by the flow sensitivity. Many experimental studies [15,20-22] have been performed with the aim of determining the optimal location of an NSDBD actuator on such a profile. These studies have consistently shown that the maximum effect is achieved when the electrodes are positioned such that the discharge zone is located just upstream of the separation point.

\subsection{Power Supply}

The core of the power supply used in the experiments is a Behlke HTS111-06GSM fast high-voltage (HV) transistor switch, composed of two identical MOSFET (metal-oxide-semiconductor field-effect transistor) switching paths forming

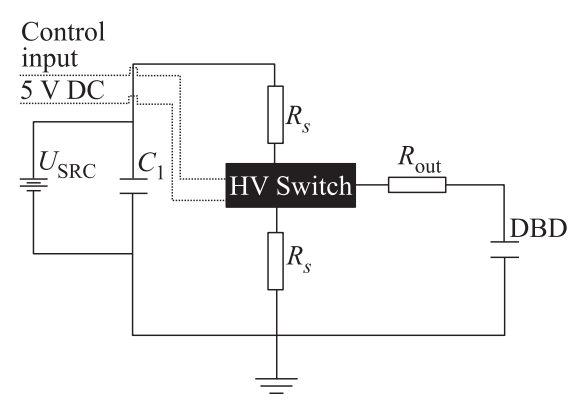

Figure 3 Power supply setup (from [17]) a so-called push-pull circuit. The basic circuit of the power supply is given in Fig. 3. A 5-volt direct current (DC) power supply and a function generator are needed to drive the switch and control the output, the latter serving as a trigger signal. A DC voltage supply provides a constant voltage of up to $10 \mathrm{kV}$, which charges the capacitor $C_{1}$ with a capacitance of $7000 \mathrm{pF}$. Once the switch opens the DBD actuator subcircuit, the voltage is primarily provided by the capacitor. Therefore,

the DC power supply can operate with a low output current. The current required to charge the capacitor depends on the repetition rate of the output voltage pulse.

The actuator was operated by means of nanosecond pulses generated by this power supply. Two operating regimes where used: one was periodic (NS-P), with a constant frequency of pulses fed into the discharge gap, and the other was a burst mode (NS-B), with pulses fed in bursts. Each pulse had a length of $200 \mathrm{~ns}$ and a peak voltage of either 3 or $6 \mathrm{kV}$. Although the peak voltage that can be provided by the power supply is $10 \mathrm{kV}$, this value was not attainable during these experiments. In the case of the burst mode signal, three pulses were used per burst and the delay between each pulse in the burst was $4 \mu \mathrm{s}-$ 


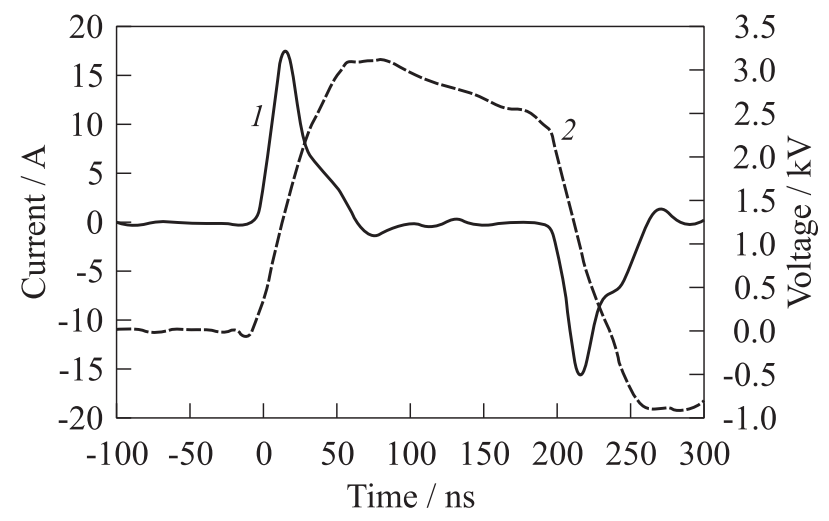

Figure 4 Sample current (1) and voltage (2) waveforms

corresponding to a frequency of $250 \mathrm{kHz}$. The current was measured by a Bergoz CT-CO-25 current transformer. Typical waveforms for voltage and current used during the experiments are shown in Fig. 4.

\subsection{Test Conditions}

In the baseline experiments where measurements were performed on the smooth profile, two values of free-stream velocity produced by the wind tunnel were investigated: 17.4 and $24 \mathrm{~m} / \mathrm{s}$ corresponding to Reynolds numbers relative to the chord of 166,000 and 230,000, respectively. The angle of attack was varied from $5^{\circ}$ to $26^{\circ}$ in steps of $1^{\circ}$. Measurements of the pressure along the model chord were recorded at all angles of attack, $\alpha$, while oil flow visualizations were performed at selected values of $\alpha$.

With the installed actuator, the experiments were performed at the same two values of Reynolds number at pre- and poststall angles of attack. In the poststall $\alpha$ range, the tested values of $\alpha$ were $15^{\circ}$ and $16^{\circ}$ up to $26^{\circ}$ in steps of $2^{\circ}$. The frequency of the pulsed signal was varied between $F_{c}^{+}=0.5$ and 15 corresponding to $f=58$ to $1740 \mathrm{~Hz}$ at $\mathrm{Re}=166,000$ and $f=80$ to $2400 \mathrm{~Hz}$ at $\mathrm{Re}=230,000$. Some measurements with an applied frequency of $10 \mathrm{kHz}$ were also performed. At each setting, two voltage levels $(3$ and $6 \mathrm{kV})$ were tested. In the prestall range, $\alpha=7^{\circ}$ at $\operatorname{Re}=166,000$ was tested with a voltage magnitude of $6 \mathrm{kV}$. For this case, the frequency was varied between $F_{c}^{+}=0.5$ and 40 corresponding to the maximum frequency of $4640 \mathrm{~Hz}$. The influence of pulse and burst mode actuation was also investigated over a smaller range of $F_{c}^{+}$in both pre- and poststall regimes.

Hot-wire anemometry and oil-flow visualization with the deactivated actuator were also performed at the selected values of $\alpha$ in both pre- and poststall regimes. 


\section{RESULTS}

\subsection{Oil-Flow Visualization}

Oil visualization of the surface flow patterns was performed with the aim to characterize the progression of separation on the airfoil as well as to locate the separation point at full stall. Two examples of the results are shown in Fig. 5 for $\operatorname{Re}=230,000$. The flow in the presented results is directed from the bottom towards the top. At prestall angles of attack, i. e., $\alpha \leq 13^{\circ}$, a separation bubble forms downstream of the leading edge of the profile with its location moving increasingly upstream as $\alpha$ is increased. The separation bubble and trailing edge separation at $\alpha=12^{\circ}$ can be observed. Figure $5 b$ shows the results at the first stall angle $\left(\alpha=14^{\circ}\right)$ where the flow is seen to separate at a distance of around 3 percent chord length from the leading edge $(x / c=0.03)$. This distance was not measured directly from the photos shown here but by visual comparison with a scale drawn on the side of the profile as the rotated profile is not captured well by the camera. The trend of the upstream movement of the separation point with increasing angle of attack is still observed in the obtained results. This upstream movement is caused by the increasingly adverse pressure gradient due to the increase in $\alpha$, inducing an earlier laminar separation. At an angle of attack of $22^{\circ}$, the separation point appears to be situated at around $1.5 \%$ of the chord length.

At a free-stream Reynolds number of 166,000, the onset of the separation bubble occurs at a slightly further upstream location when compared to the corresponding case at $\mathrm{Re}=230,000$. The separation resistance of a boundary layer increases with Reynolds number [24]; so, it is to be expected that at the lower value of Re, the boundary layer separates earlier. However, the difference

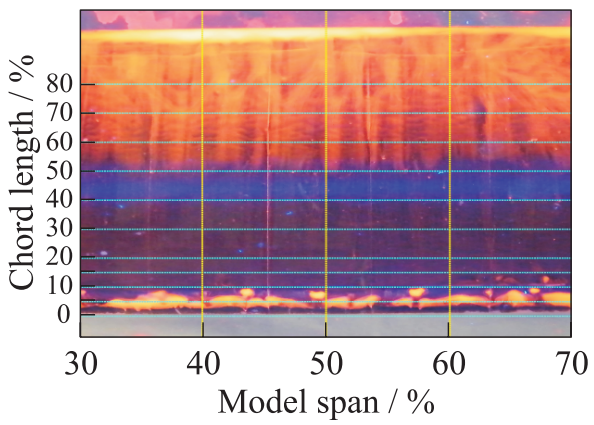

(a)

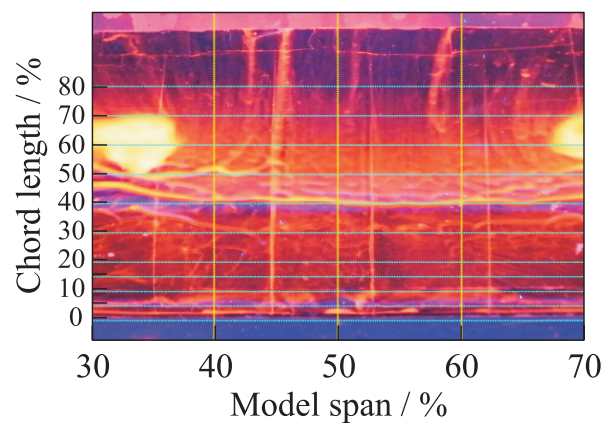

(b)

Figure 5 Pre- $(a)$ and poststall $(b)$ flow visualization results at $\mathrm{Re}=230,000$ for $\alpha=12^{\circ}(a)$ and $14^{\circ}(b)$ 


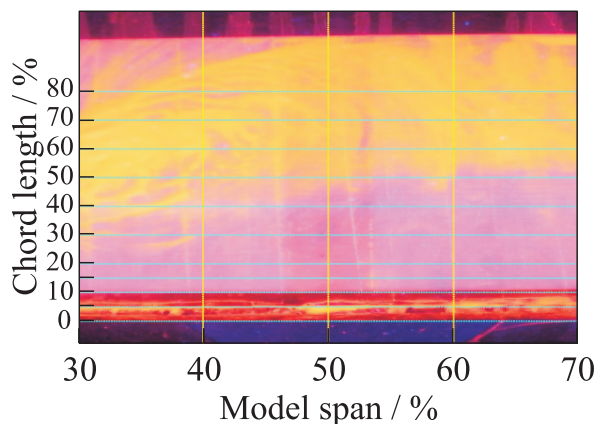

(a)

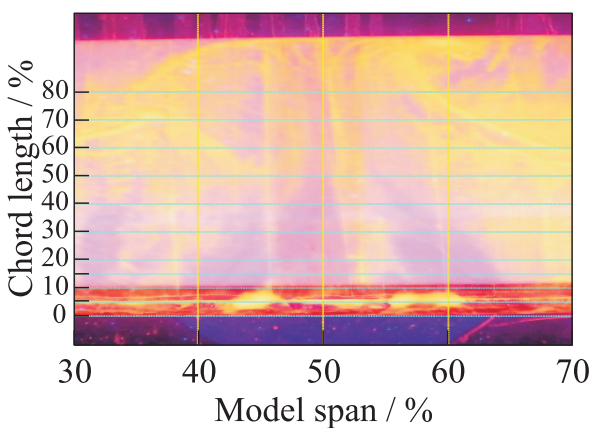

(b)

Figure 6 Pre- $(a)$ and poststall $(b)$ flow visualization results at $\operatorname{Re}=230,000$ (with installed actuator) for $\alpha=14^{\circ}(a)$ and $16^{\circ}(b)$

between the tested values of Re is not large enough to cause a significant change in behavior in the two cases.

Oil-flow visualization was also performed for the airfoil with the installed but deactivated actuator in order to investigate the influence of the actuator geometry itself on the flow. Differences in the separation bubble were observed between these results and those obtained with the smooth model. The presence of the exposed electrode forms a forward facing step which induces an earlier laminar separation, moving the separation bubble upstream of the position observed in the corresponding smooth airfoil results. It can be also noted that there are variations in the separation bubble over the model span; some regions accumulate more oil than others. This is most likely due to irregularities present on the exposed electrode which was cut and attached to the model by hand. Figure $6 a$ shows that attached flow is obtained at $\alpha=14^{\circ}$ as opposed to the smooth model. The presence of the exposed electrode induces an earlier laminar-toturbulent transition, improving the boundary layer's separation resistance close to the model's leading edge. At the onset of stall, the separation point remains at a similar location to that observed on the smooth airfoil as seen in Fig. $6 b$.

\subsection{Hot-Wire Anemometry}

Hot-wire measurements were performed at a vertical distance of $5 \mathrm{~mm}$ over the profile at $x / c=0.7$ with the aim of distinguishing dominant frequencies in the flow. The results of the measurements taken over the profile are given in Fig. 7, at $\alpha$ values in both pre- and poststall regimes. The power spectral density (PSD) describes how the power of a signal is distributed over different frequencies and was calculated by Welch's method. A peak in the PSD value 


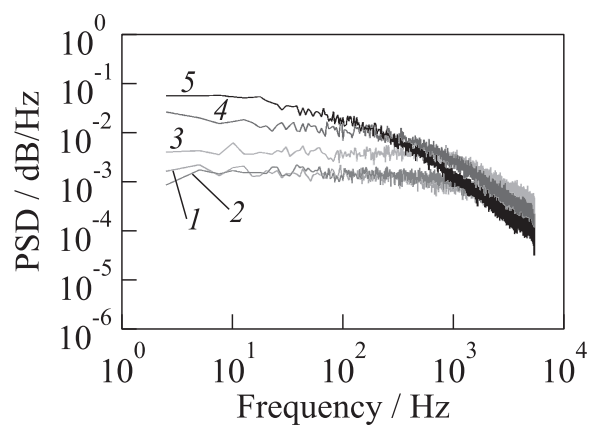

(a)

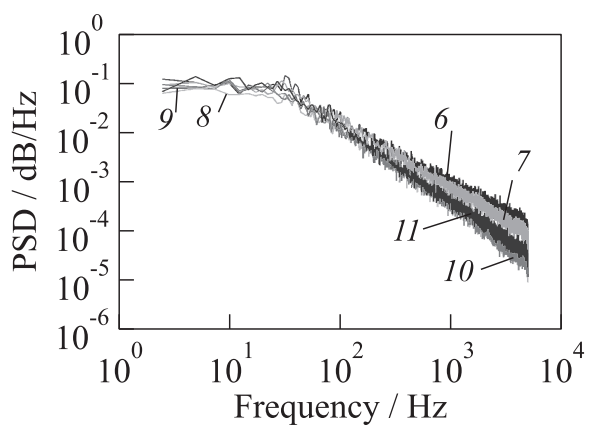

(a)

Figure 7 Spectra of velocity pulsations measured at an offset of $5 \mathrm{~mm}$ over the profile at $x / c=0.7, \operatorname{Re}=230,000$ in pre- $(a)$ and poststall $(b)$ regimes: $1-\alpha=3^{\circ}$, $\mathrm{Tu}=7.1 \% ; 2-\alpha=5^{\circ}, \mathrm{Tu}=7.4 \% ; 3-\alpha=7^{\circ}, \mathrm{Tu}=15 \% ; 4-\alpha=9^{\circ}, \mathrm{Tu}=31.2 \%$; $5-\alpha=13^{\circ}, \mathrm{Tu}=45.7 \% ; 6-\alpha=15^{\circ}, \mathrm{Tu}=35.6 \% ; 7-\alpha=17^{\circ}, \mathrm{Tu}=41.9 \%$; $8-\alpha=19^{\circ}, \mathrm{Tu}=41.5 \% ; 9-\alpha=21^{\circ}, \mathrm{Tu}=41.9 \% ; 10-\alpha=23^{\circ}, \mathrm{Tu}=43.9 \%$; and $11-\alpha=25^{\circ}, \mathrm{Tu}=48.2 \%$

at a certain frequency would indicate that this particular frequency is dominant in the flow. Here, the aim was to investigate whether operation of the NS-DBD actuator at that frequency would produce any significant results.

The turbulence level in the measurements increases as the angle of attack increases up to $13^{\circ}$ showing that the laminar-turbulent transition occurs further and further upstream as $\alpha$ increases. Between $13^{\circ}$ and $15^{\circ}$, the turbulence level decreases; this is where the flow changes from partially attached to fully separated. The energy shed in the flow oscillations also increases with $\alpha$. However, at this measurement location, no dominant frequencies could be identified in the flow. It is likely that more interesting results could be obtained if the hot wire measurements were performed closer to the leading edge, though this was not possible due to the insufficient length of the hot wire probe. Also, in such situations, there is a danger of too many EMI (electromagnetic interferences), that render difficult the interpretation.

\subsection{Activation of the Nanosecond Dielectric Barrier Discharge Actuator: Poststall Results}

As described in subsection 2.1, only incomplete pressure profiles could be measured due to application of the DBD actuator on the leading edge of the profile. Hence, the lift and drag coefficients are influenced as this is a critical region for their evaluation and so could not be evaluated precisely. Hence, a different 


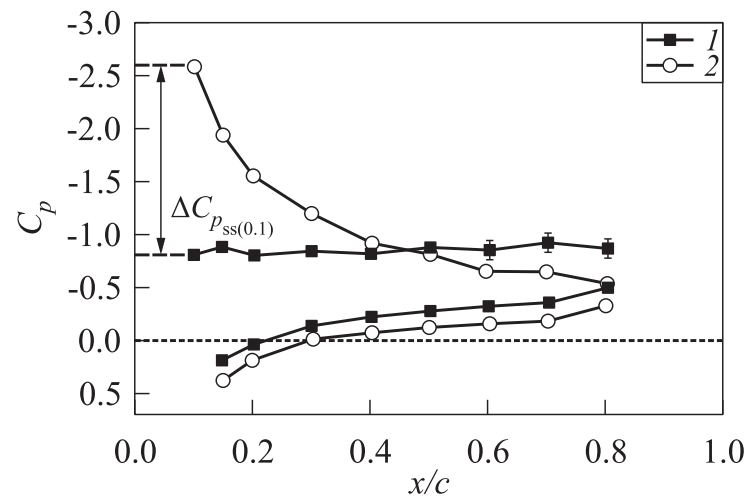

Figure 8 Estimation of the value of $\left|\Delta C_{p_{\mathrm{ss}(0.1)}}\right|: \quad 1$ - plasma off and 2 $f=0.116 \mathrm{kHz}\left(F_{c}^{+}=1\right), \mathrm{NS}-\mathrm{P}$

characteristic of the flow was used to quantify the influence of the discharge: the increase in lift induced by the discharge was approximated by the absolute difference in the pressure coefficient $C_{p}$ at $x / c=0.1$ on the suction side of the profile measured with and without plasma. This $\left|\Delta C_{p_{\mathrm{ss}(0.1)}}\right|$ was calculated as shown in Fig. 8. By observing the pressure profiles in Fig. 9, it can be seen that this approximation for the $\Delta C_{L}$ is quite intuitive, as the greatest difference in the pressure profile induced by the actuator is observed at this location. The larger

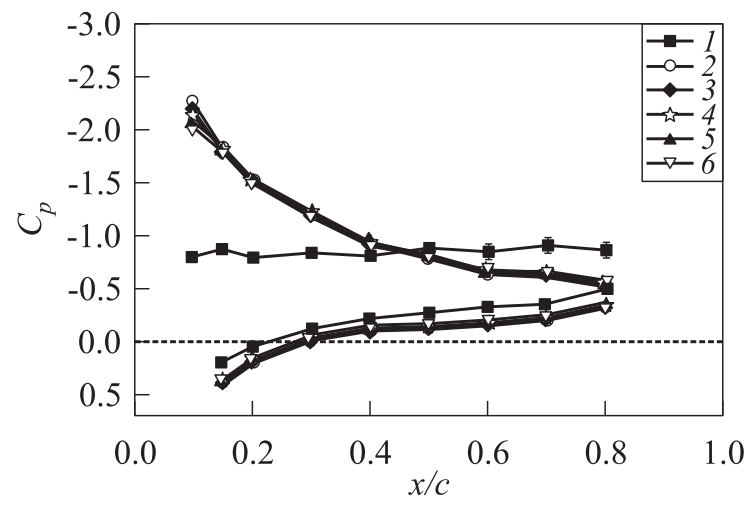

Figure 9 Typical obtained pressure profiles $\left(\alpha=16^{\circ}, U=6 \mathrm{kV}\right.$, and $\left.\operatorname{Re}=166,000\right)$ : 1 - plasma off; $2-f=0.348 \mathrm{kHz}\left(F_{c}^{+}=3\right)$, NS-P; $3-f=0.464 \mathrm{kHz}\left(F_{c}^{+}=4\right)$, NS-P; $4-f=0.580 \mathrm{kHz}\left(F_{c}^{+}=5\right)$, NS-P; $5-f=0.696 \mathrm{kHz}\left(F_{c}^{+}=6\right)$, NS-P; and $6-f=0.812 \mathrm{kHz}\left(F_{c}^{+}=7\right)$, NS-P 
the observed value of $\left|\Delta C_{p_{\mathrm{ss}(0.1)}}\right|$, the more effective the plasma is deemed to be. The plots shown all include error bars that represent the standard deviation of the variable in question.

\section{Influence of applied voltage}

Two values of voltage ( 3 and $6 \mathrm{kV}$ ) were used to create a plasma with the actuator in order to investigate the effect of plasma strength on flow attachment. The threshold voltage for the creation of plasma is approximately $2.5 \mathrm{kV}$, implying that the 3-kilovolt plasma is rather weak. Nevertheless, this voltage level was still found to have a significant impact on the flow. The results obtained for the angles of attack only slightly larger than the stall angle, $15^{\circ}$ and $16^{\circ}$, at both applied voltage levels, were quite similar. Figure $10 a$ shows the pressure profiles at a Reynolds number of 166,000 and at the value of reduced forcing frequency $F_{c}^{+}$that generates the highest lift for the both $\alpha$ values. This is discussed further in subsection 3.4. Interestingly, the difference between the two applied voltages on the flow is not significant, whereas one would expect that a stronger plasma should have a greater influence on the flow. In fact, the indication is that the lower voltage is slightly more effective. Similar results were obtained with $\operatorname{Re}=230,000$.

This trend is continued at higher frequency values where the results in Fig. $11 a$ again show that the 3-kilovolt plasma has a greater influence on the flow than the 6-kilovolt. The $\left|\Delta C_{p_{\mathrm{ss}(0.1)}}\right|$ achieved with the 3-kilovolt plasma is greater than the 6-kilovolt plasma, and the results are consistent for increasing values of $F_{c}^{+}$as can be seen in Fig. 11a. Similar results were obtained for $\alpha=15^{\circ}$. As the angle of attack is increased to $18^{\circ}$ and beyond, the magnitude of the applied voltage starts to have a critical effect on the plasma's influence on

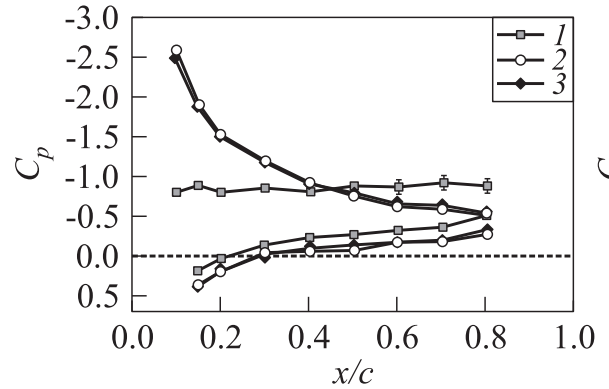

(a)

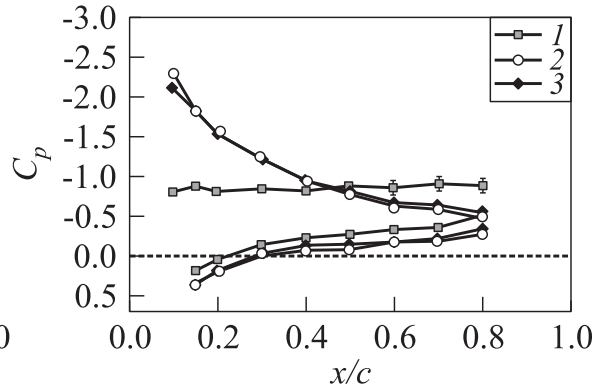

(b)

Figure 10 Effect of NS-DBD pulsed actuator on the pressure profiles at $\alpha=16^{\circ}$, $\mathrm{Re}=166,000, F_{c}^{+}=1(a)$ and $5(b)$, and different applied voltages: 1 - plasma off; $2-U=3 \mathrm{kV}, \mathrm{NS}-\mathrm{P}$; and $3-U=6 \mathrm{kV}, \mathrm{NS}-\mathrm{P}$ 


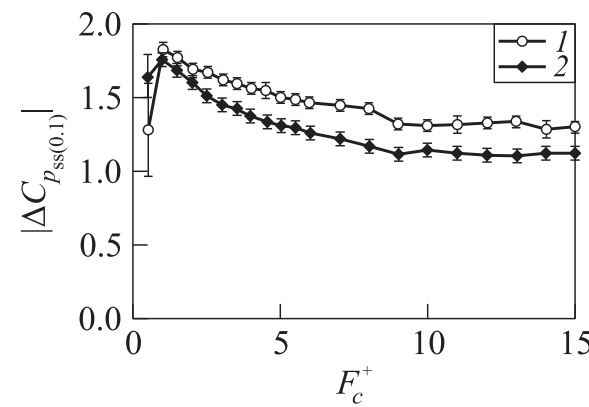

(a)

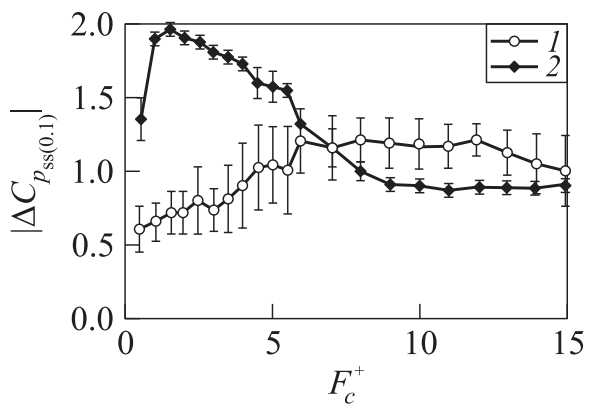

(b)

Figure 11 Effect of the NS-DBD pulsed actuator operated at different applied voltages $(1-U=3 \mathrm{kV}$ and $2-U=6 \mathrm{kV})$ on the $\left|\Delta C_{p_{\mathrm{ss}(0.1)}}\right|$ value over the range of tested $F_{c}^{+}$values: $(a) \alpha=16^{\circ}, \operatorname{Re}=166,000 ;$ and $(b) \alpha=18^{\circ}, \operatorname{Re}=230,000$

the flow. Figure $11 b$ shows that the 6 -kilovolt plasma now attaches the flow more effectively than the 3-kilovolt plasma. The error bars for the 3-kilovolt plasma results are also quite large, indicating that the suction side pressure distribution is not as steady as that obtained with the 6-kilovolt plasma. The energy delivered to the flow by the 3-kilovolt plasma no longer seems to be large enough to induce full attachment, leading to a smaller value of $\left|\Delta C_{p_{\mathrm{ss}(0.1)}}\right|$ and larger error bars over the tested frequency range. The observed trend is that an increasing plasma strength is required to effectively attach the flow as the angle of attack is increased.

The reason why the 3 -kilovolt plasma is more effective at $\alpha=15^{\circ}$ and $16^{\circ}$ is not intuitive and this shows that there is more to the mechanism with which the plasma actuator influences the flow than influencing and enhancing a laminar to turbulent tripping and existing turbulence enhancement within the boundary layer. A minimum energy threshold for flow attachment seems to exist at which the peak plasma effectiveness is achieved. Increasing the energy beyond this value either by increasing the applied voltage or by operating in burst mode (as will be discussed in subsection 3.4) results in a decrease in actuator performance. The obtained results have also shown an increasing energy requirement for flow attachment as the angle of attack increases. The reason for this could be twofold: first, an increasing amount of energy might be required to effectively excite the shedding structures which entrain the flow in the boundary layer and induce attachment at larger angles of attack. Another reason could be the upstream movement of the separation line with increasing $\alpha$ as observed in the oil-flow visualization experiments. If the actuator is no longer at the optimal position, the larger voltage might need to be applied in order to deliver the required energy at the point on the actuator which corresponds to the new separation location. 


\subsection{Influence of Applied Frequency}

As can already be noted from the results presented as far, the frequency at which the NS-DBD actuator is pulsed can have a significant effect on the development of the flow over the profile. Comparing with other experimental works $[7,16]$, it was expected that the maximum effect of the plasma on the flow would be obtained in the region of $F_{c}^{+}=1-2$. The experimental results obtained do in fact follow this trend; Fig. 12 shows the value of $\left|\Delta C_{p_{\mathrm{ss}(0.1)}}\right|$ plotted against $F_{c}^{+}$ for all tested angles of attack and Reynolds numbers for $U=6 \mathrm{kV}$. It can be seen that the peak $\left|\Delta C_{p_{\mathrm{ss}(0.1)}}\right|$ value is reached in the range of $F_{c}^{+}=1-1.5$ at least for $\alpha=15^{\circ}$ and $16^{\circ}$ at both Reynolds numbers and for $\alpha=18^{\circ}$ and $\operatorname{Re}=230,000$. For the other $\alpha$ values, the peak $\left|\Delta C_{p_{\mathrm{ss}(0.1)}}\right|$ is either shifted to higher values of frequency, e.g., $F_{c}^{+}=5$ in the $\alpha=20^{\circ}$ case, or a broad peak over $F_{c}^{+} \leq 4$ occurs.

For $\alpha$ values up to $20^{\circ}$, the plasma induced a change in the flow at all tested $F_{c}^{+}$values whereas for the higher angles of attack, the plasma influence decreased to zero at $F_{c}^{+} \geq 5$ at $\operatorname{Re}=166,000$ and $F_{c}^{+} \geq 3$ at $\operatorname{Re}=230,000$. It is likely that as $F_{c}^{+}$increases beyond 15 for $\alpha \leq 20^{\circ}$, the influence of the plasma would also decrease until it is no longer effective. In fact, some additional measurements were performed with $f=10 \mathrm{kHz}$ which corresponds to $F_{c}^{+}=86.2$ at Re $=166,000$ and $F_{c}^{+}=62.5$ at $\operatorname{Re}=230,000$. With this frequency setting and values of $\alpha=16^{\circ}$ and $U=6 \mathrm{kV}$, the actuator had little, if any, influence on the flow.

The plots presented in Fig. 12 show that the peak $\left|\Delta C_{p_{\mathrm{ss}(0.1)}}\right|$ occurs in the range of $F_{c}^{+}=1-1.5$ for those cases where the plasma energy seems to be above the minimum threshold required for effective operation of the actuator. These

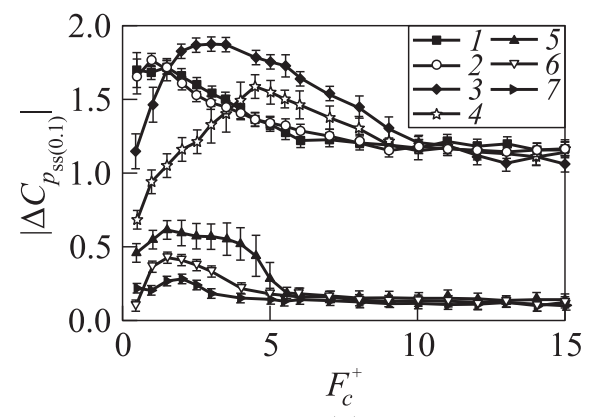

(a)

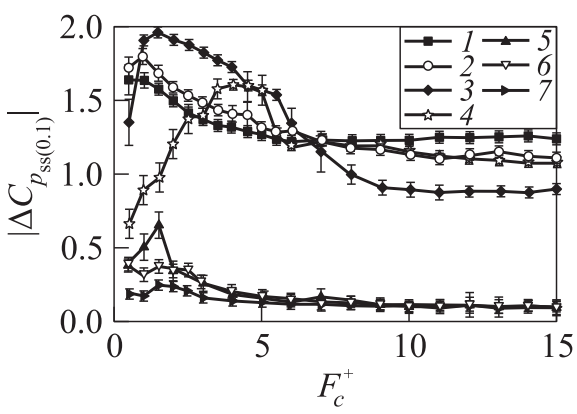

(b)

Figure 12 Effect of the NS-DBD pulsed actuator at $U=6 \mathrm{kV}$ and different angles of attack $\left(1-\alpha=15^{\circ} ; 2-16^{\circ} ; 3-18^{\circ} ; 4-20^{\circ} ; 5-22^{\circ} ; 6-24^{\circ}\right.$; and $7-$ $\left.\alpha=26^{\circ}\right)$ on the $\left|\Delta C_{p_{\mathrm{ss}(0.1)}}\right|$ value over the range of tested $F_{c}^{+}$values: $(a) \operatorname{Re}=166,000$; and $(b) \operatorname{Re}=233,000$ 
are the cases where $\alpha=15^{\circ}$ and $16^{\circ}$ at both Reynolds numbers and $\alpha=18^{\circ}$ at $\operatorname{Re}=230,000$. Within this $F_{c}^{+}$range, it is likely that the dominant mechanism of the plasma influence is the excitation of shedding structures over the profile which results in flow entrainment and attachment. Unfortunately, the presence of these vortices could not yet be confirmed experimentally. This will be the subject of upcoming test campaigns [25].

At higher values of $F_{c}^{+}$and at the cases where the plasma energy was deemed to be too small to produce the maximum possible flow attachment by vortex excitation, the plasma was still observed to have an effect. At high frequencies, the plasma likely acts as a boundary layer tripping device through the generation of thermal compression waves. This mechanism of operation could not be confirmed via pressure tap measurements, and is completed by PIV measurements in a follow-up work [25]. If the applied voltage was increased to provide the minimum required energy, $F_{c}^{+}$with the maximum effect on the flow reduces to the range of $1-1.5$ observed for the other cases.

\subsubsection{Experimental observations}

The presented results show that for $\alpha \leq 22^{\circ}$, the generated plasma always has an influence on the flow causing at least some partial attachment close to the leading edge for the range of tested $F_{c}^{+}$. Interestingly, the value of $F_{c}^{+}$had an influence on the ease with which the flow attached, i. e., some values of $F_{c}^{+}$had an immediate effect on the flow once the plasma was switched on, whereas other values had a less prompt effect. Typically, the values of $F_{c}^{+} \leq 6$ induced an immediate attachment close to the leading edge, while the higher values would require some time (typically, more than $1 \mathrm{~min}$ ) before having an effect on the flow. This observation again indicates that at the tested conditions, the values of $F_{c}^{+}$in the range of 1 to 1.5 are more effective for flow control.

\subsubsection{Influence of applied actuation signal}

Figure 13 shows the pressure profiles at different values of $\alpha$, for the 3 - and 6-kilovolt applied voltages, operated in both pulse and burst modes. Two different types of behavior can be observed in the results obtained: at $\alpha=15^{\circ}$ and $16^{\circ}$, where the burst mode is less effective than the pulse mode, and at $\alpha \geq 18^{\circ}$, where the burst mode is more effective than the pulse mode. These trends are consistent in all the tested cases as can be seen in Fig. 14. In fact, for the $\alpha=18^{\circ}$ case, the peak $\left|\Delta C_{p_{\mathrm{ss}(0.1)}}\right|$ is shifted and now occurs around $F_{c}^{+}=1$ range, which is around the value expected for maximum plasma effectiveness. Similarly at $\alpha=20^{\circ}$, the peak $\left|\Delta C_{p_{\mathrm{ss}(0.1)} \mid}\right|$ is shifted closer to the optimal range of $F_{c}^{+}$but does not reach it. It is likely that additional energy input is required for the plasma to have the maximum effect. 


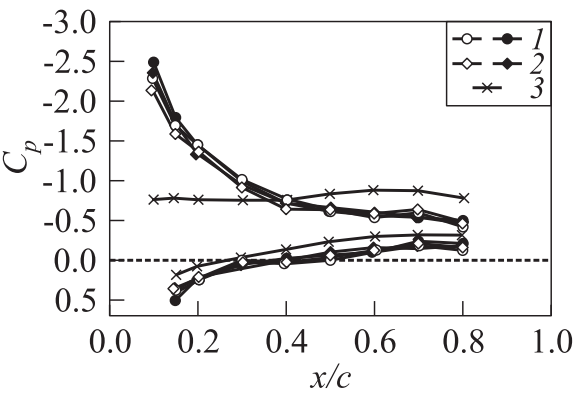

(a)

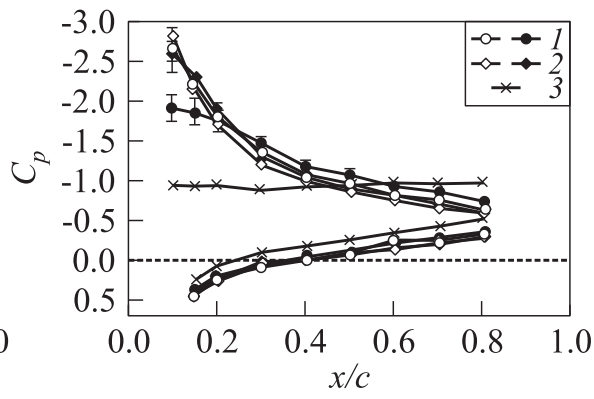

(b)

Figure 13 Effect of the NS-DBD actuator operated in pulsed (filled signs) and burst (empty signs) modes at different applied voltages $(1-3 \mathrm{kV} ; 2-6 \mathrm{kV}$; and $3-$ plasma off) on the pressure profiles at $\operatorname{Re}=166,000:(a) \alpha=15^{\circ}, F_{c}^{+}=2.5$; and (b) $\alpha=18^{\circ}, F_{c}^{+}=1.5$

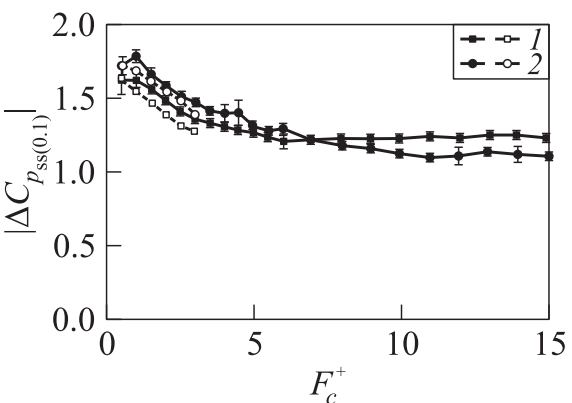

(a)

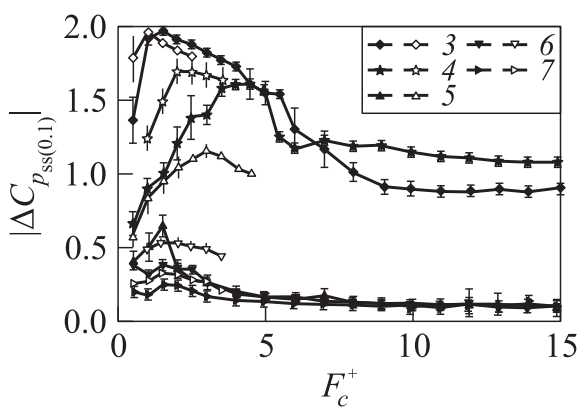

(b)

Figure 14 Effect of the NS-DBD pulsed actuator operated in pulsed (filled signs) and burst (empty signs) modes on the $\left|\Delta C_{p_{\mathrm{ss}(0.1)}}\right|$ value over the range of tested $F_{c}^{+}$ values at $\mathrm{Re}=230,000, U=3(a)$ and $6 \mathrm{kV}(b)$, and different angles of attack: 1 $\alpha=15^{\circ} ; 2-16^{\circ} ; 3-18^{\circ} ; 4-20^{\circ} ; 5-22^{\circ} ; 6-24^{\circ}$; and $7-\alpha=26^{\circ}$

These results can be compared with those presented in subsection 3.3, where the influence of applied voltage was discussed. At $\alpha=15^{\circ}$ and $16^{\circ}$, the peak $\left|\Delta C_{p_{\mathrm{ss}(0.1)}}\right|$ is obtained at an applied voltage of $3 \mathrm{kV}$. As the energy supplied to the boundary layer is increased either by increasing the number of pulses in a burst or by increasing the applied voltage, the effectiveness of the actuator is reduced. As the angle of attack is increased to $18^{\circ}$ and above, the 3-kilovolt pulsed plasma still affects the boundary layer and induces some attachment; however, the 3-kilovolt burst mode is more effective than the 3-kilovolt pulse mode. The 6-kilovolt pulse mode, in turn, has a greater influence on the flow 
than the 3-kilovolt burst case, but the 6-kilovolt burst mode is still more effective. These results again indicate that the maximum plasma effectiveness occurs once the minimum energy required to excite coherent structures in the boundary layer is reached. Increasing beyond this value results in the reduced effect on the flow, similar to when a certain minimum energy level is not reached. Hence, there is a distinct energy zone for the actuation to be effective.

\subsubsection{Influence of angle of attack}

The trends that are apparent with increasing angle of attack have already been discussed in the previous sections. Thus, no additional plots are presented. With an increasing angle of attack, additional energy input is required to maximize the effect of the plasma on the flow. This could be due either to the movement of the separation line or, else, to the increase in the energy required to successfully excite the vortices over the profile. The value of $F_{c}^{+}$which maximizes the effect of the plasma on the flow remains in the range of $1-1.5$ as the angle of attack is increased provided that the supplied energy is greater than or equal to the threshold requirement.

\subsection{Activation of the Nanosecond Dielectric Barrier Discharge Actuator: Prestall Results}

A separation bubble was observed on the profile as early as $\alpha=7^{\circ}$ and $\operatorname{Re}$ $=166,000$ up to $x / c=0.2$, as can be seen from the flat portion of the pressure

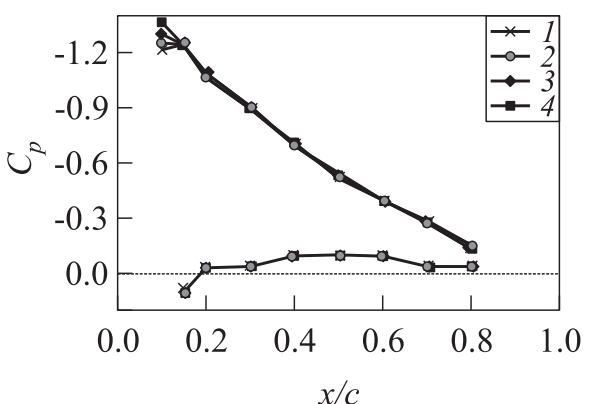

(a)

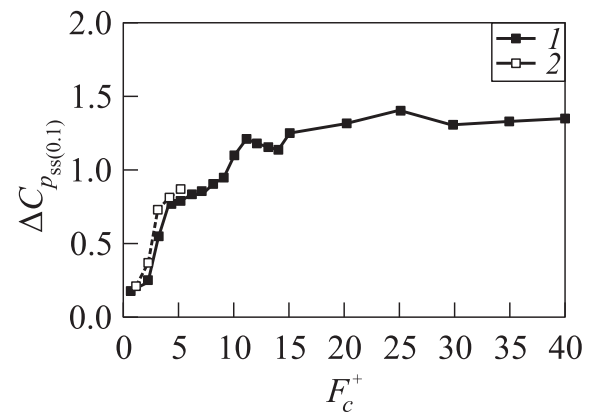

(b)

Figure 15 Effect of the NS-DBD actuator operated in pulsed (filled signs) and burst (empty signs) modes with $U=6 \mathrm{kV}$ on the separation bubble observed at $\alpha=7^{\circ}$ and $\operatorname{Re}=166,000:(a)$ selected pressure profiles: $1-$ plasma off; $2-f=0.232 \mathrm{kHz}$ $\left(F_{c}^{+}=2\right) ; 3-f=0.812 \mathrm{kHz}\left(F_{c}^{+}=7\right)$; and $4-f=4.64 \mathrm{kHz}\left(F_{c}^{+}=40\right)$; and (b) variation of the $\left|\Delta C_{p_{\mathrm{ss}(0.1)}}\right|$ with $F_{c}^{+}$and signal type mode 
profile (without plasma) in Fig. 15a. This indicates that in this case, the activated actuator works as an active tripping device, inducing an earlier and quicker laminar-to-turbulent transition and effectively eliminating the separation bubble over that area of the profile. Figure $15 b$ plots the $\left|\Delta C_{p_{\mathrm{ss}(0.1)}}\right|$ with $F_{c}^{+}$, with the maximum $F_{c}^{+}=40$ (note that here, the $y$-axis scale is different from the similar plots presented previously and that errorbars are not included for clarity). The actuator effectiveness increases with frequency until $F_{c}^{+}=10$, after which it remains more or less constant until $F_{c}^{+}=40$.

\section{CONCLUDING REMARKS}

In this paper, the influence of a pulse surface NS-DBD actuator on the flow around an airfoil at pre- and poststall angles of attack has been investigated for free-stream velocities up to $24 \mathrm{~m} / \mathrm{s}$. The ability of the NS-DBD actuator to delay stall by controlling flow separation from the leading edge of the airfoil has been clearly proven for angles of attack up to $\alpha=22^{\circ}$; an increase of $8^{\circ}$ over the no plasma case. An influence of the activated NS-DBD actuator on the flow was observed at all tested angles of attack (up to $26^{\circ}$ ); and based on the results obtained, it is likely that the stall angle could be extended further if the actuator settings were to be optimized accordingly.

The influence of different NS-DBD actuator settings, i. e., applied voltage, frequency, and signal type on the flow attachment has been investigated together with variations in the model's angle of attack and the free-stream flow velocity. The obtained results have shown that there seem to be two different mechanisms at work in the plasma-flow interaction: one which is active over a wide range of frequencies and another which greatly enhances the effectiveness of the actuator around $F_{c}^{+}=1$. Indeed, these observed effects seem to correspond with the two mechanisms of plasma-flow interaction commonly described in literature: enhancement of boundary layer turbulence at high frequencies and excitation of boundary layer shedding structures close to $F_{c}^{+}=1$. The occurrence of these shedding structures induces a more effective attachment of the flow. However, the results show that there exists a minimum energy requirement to excite these structures which is different at each flow condition. This energy must be delivered to the flow in order to induce maximum flow attachment. Interestingly, for those cases close to the stall angle where the smallest power setting on the actuator $\left(3 \mathrm{kV}\right.$, NS-P mode) induced maximum attachment at $F_{c}^{+}=1$ to 1.5 , increasing the power beyond this value consistently had a detrimental impact on the flow attachment.

As the angle of attack was increased away from the stall angle, additional power was required to effectively induce flow attachment. Possible reasons for this could include a greater energy requirement to excite flow instabilities or 
simply that the separation location has changed and that the actuator is no longer at the optimal location.

An interesting perspective is the observation of the boundary layer velocity field and consequent the shedding structures and vortices using PIV measurements that are currently under progress [25]. A more complete investigation of free-stream laminarity also needs to be evaluated to understand the tripping mechanism or not of the plasma excitation.

In conclusion, the work presented here provides evidence that NS-DBD can be used as a flow control actuator for separation delay and the optimal reduced frequencies are those around the value of 1 .

\section{REFERENCES}

1. Prandtl, L. 1904. Über Flüssigkeitsbewegung bei sehr kleiner Reibung. 3rd Math. Congress (International) Proceedings. Heidelberg, Germany. 484-491.

2. Lachmann, G. V. 1961. Boundary layer and flow control. Its principles and application. New York: Pergamon Press. Vol. 1. 612 p.

3. Gad-el-Hak, M. 1996. Modern developmenst in flow control. Appl. Mech. Rev. 49:365-379.

4. McLean, J. D., J. D. Crouch, R. C. Stoner, S. Sakurai, G. E. Seidel, W. M. Feifel, and H. M. Rush. 1999. Study of the application of separation control by unsteady excitation to civil transport aircraft. Technical Report NASA CR-1999-209228.

5. Crowther, W. J. 2006. Separation control on a trailing-edge flap using air jet vortex generators. J. Aircraft 43(5):1589-1593.

6. Wilkinson, S. P. 1990. Interactive wall turbulence control. In: Viscous drag reduction in boundary layers. Eds. D. M. Bushnell and J. N. Hefner. Progress in astronautics and aeronautics ser. Washington, D.C.: AIAA. 123:479-509.

7. Seifert, A., A. Darabi, and I. Wygnanski. 1996. Delay of airfoil stall by periodic excitation. J. Aircraft 33:691-698.

8. Cattafesta, L. N., and M. Sheplak. 2011. Actuators for active flow control. Annu. Rev. Fluid Mech. 43:247-272.

9. Corke, T. C., C. Lon Enloe, and S. P. Wilkinson. 2010. Dielectric barrier discharge plasma actuators for flow control. Annu. Rev. Fluid Mech. 42:505-529.

10. Erfani, R., T. Erfani, C. Hale, K. Kontis, and S. V. Utyzhnikov. 2011. Optimization of induced velocity for plasma actuator with multiple encapsulated electrodes using response surface methodology. AIAA Paper No. 2011-1206.

11. Caruana, D., C. Tropea, Ch. Hollenstein, J.-P. Boeuf, and K. S. Choi, eds. 2013. PLASMAERO European Project. ERCOFTAC Special Theme: Plasma Aerodynamics. 94.

12. Moreau, E. 2007. Airflow control by non-thermal plasma actuators. J. Phys. D Appl. Phys. 40:605-636. 
13. Starikovskii, A. Yu., A. A. Nikipelov, M. M. Nudnova, and D. V. Roupassov. 2009. SDBD plasma actuator with nanosecond pulse-periodic discharge. Plasma Sources Sci. Technol. 18(3):034015.

14. Patel, M. P., T. T. Ng, S. Vasudevan, M. L. Post, T. E. McLaughlin, and C. F. Suchomel. 2008. Scaling effects of an aerodynamic plasma actuator. J. Aircraft 45:223-236.

15. Roupassov, D. V., A. A. Nikipelov, M. M. Nudnova, and A. Yu. Starikovskii. 2009. Flow separation control by plasma actuator with nanosecond pulsed-periodic discharge. AIAA J. 47:168-185.

16. Rethmel, C., J. Little, K. Takashima, A. Sinha, I. Adamovich, and M. Samimy. 2011. Separation control with nanosecond-pulse-driven dielectric barrier discharge plasma actuators. AIAA Paper No. 2011-487.

17. Peschke, P., S. Goekce, Ch. Hollenstein, P. Leyland, and P. Ott. 2011. Interaction between nanosecond pulse DBD actuators and transonic flow. AIAA Paper No. 2011-3734.

18. Marino, A., P. Peschke, F. De Gregorio, P. Leyland, P. Ott, Ch. Hollenstein, and R. S. Donelli. 2013. High voltage pulsed DBD effects on the aerodynamic performances and on the shock buffet. ERCOFTAC Special Theme: Plasma Aerodynamics 94:70-76.

19. Little, J., K. Takashima, M. Nishihara, I. Adamovich, and M. Samimy. 2012. Separation control with nanosecond-pulse-driven dielectric barrier discharge plasma actuators. AIAA J. 50:350-365.

20. Johnson, G. A., and S. J. Scott. 2001. Plasma-aerodynamic boundary layer interaction studies. AIAA Paper No. 2001-3052.

21. Sidorenko, A. A., B. Y. Zanin, B. V. Postnikov, A. D. Budovksky, A. Y. Starikovskii, D. V. Roupassov, I. N. Zavialov, N. D. Malmuth, P. Smereczniak, and J. S. Silkey. 2007. Pulsed discharge actuators for rectangular wing separation control. AIAA Paper No. 2007-941.

22. Grech, N. 2013. Investigation of flow separation control by nanosecond pulsed dielectric barrier discharge actuators at low flow speeds. Ecole Polytechnique Fédérale de Lausanne. M.Sc. Thesis.

23. Peschke, P. 2014. Experimental investigation of pulsed DBD plasma actuators for aerodynamic flow control. Ecole Polytechnique Fédérale de Lausanne. Ph.D. Thesis.

24. Lissaman, P. B.S. 1983. Low Reynolds number airfoils. Annu. Rev. Fluid Mech. 15:223-239.

25. Geuns, R. 2014. The use of dielectric barrier discharge plasma actuators for lowspeed control: An experimental study on plasma characteristics. TU Delft and Ecole Polytechnique Fédérale de Lausanne. M.Sc. Thesis. 108 p. 